\title{
Impulsive boundary value problems containing Caputo fractional derivative of a function with respect to another function
}

Chanon Promsakon', Eakachai Suntonsinsoungvon ${ }^{2}$, Sotiris K. Ntouyas ${ }^{3,4}$ and Jessada Tariboon ${ }^{1 *}$ (c)

"Correspondence:

jessada.t@sci.kmutnb.ac.th

${ }^{1}$ Intelligent and Nonlinear Dynamic

Innovations Research Center,

Department of Mathematics,

Faculty of Applied Science, King

Mongkut's University of Technology

North Bangkok, Bangkok, Thailand

Full list of author information is

available at the end of the article

\begin{abstract}
In this paper, we study the existence and uniqueness for a new class of impulsive fractional boundary value problems with separated boundary conditions containing the Caputo fractional derivative of a function with respect to another function. The existence of solutions is established by using the Leray-Schauder nonlinear alternative, and the uniqueness result is proved via Banach's contraction mapping principle. Some examples are also constructed to demonstrate the application of main results.
\end{abstract}

MSC: $34 \mathrm{~A} 08 ; 34 \mathrm{~A} 37$

Keywords: Impulsive fractional differential equations; Separated boundary conditions; Fixed point theorems

\section{Introduction and preliminaries}

Impulsive boundary value problems corresponding to integer-order differential equations with impulsive conditions have been considered extensively in the literature; see [1-3] and references therein. Also, in the case of noninteger-order differential equations, there are many results on impulsive boundary value problems; see, for example, [4-8], to name a few, but some problems still need further investigation under impulsive conditions. To the best of our knowledge, there are no papers on impulsive fractional differential equations containing the fractional derivative of a function with respect to another function. This gap is covered by the present paper.

More precisely, the purpose of this investigation is to establish the existence and uniqueness of solutions for a new class of impulsive fractional differential equations with boundary conditions of the form

$$
\left\{\begin{array}{l}
t_{k} D_{g_{k}}^{\alpha_{k}} x(t)=f(t, x(t)), \quad t \neq t_{k}, k=0,1,2, \ldots, m, \\
x\left(t_{k}^{+}\right)-x\left(t_{k}^{-}\right)=\phi_{k}\left(x\left(t_{k}\right)\right), \quad k=1,2, \ldots, m, \\
D_{g_{k}} x\left(t_{k}^{+}\right)-D_{g_{k-1}} x\left(t_{k}^{-}\right)=\phi_{k}^{*}\left(x\left(t_{k}\right)\right), \quad k=1,2, \ldots, m, \\
x(0)+\beta_{1} D_{g_{0}} x(0)=\lambda_{1}, \quad x(T)+\beta_{2} D_{g_{m}} x(T)=\lambda_{2},
\end{array}\right.
$$

(c) The Author(s) 2019. This article is distributed under the terms of the Creative Commons Attribution 4.0 International License (http://creativecommons.org/licenses/by/4.0/), which permits unrestricted use, distribution, and reproduction in any medium, provided you give appropriate credit to the original author(s) and the source, provide a link to the Creative Commons license, and indicate if changes were made. 
where ${ }_{t_{k}} D_{g_{k}}^{\alpha_{k}}$ is the Caputo-type fractional differential operator with respect to another increasing differentiable function $g_{k}(t), t \in J_{k}$, of order $1<\alpha_{k} \leq 2, J_{k}=\left(t_{k}, t_{k+1}\right]$, $k=0,1,2, \ldots, m, t_{0}=0<t_{1}<t_{2}<\cdots<t_{m}<t_{m+1}=T$ are impulsive points, the integer order of the differential operator $D_{g_{k}}$ is defined by

$$
D_{g_{k}}=\frac{1}{g_{k}^{\prime}(t)} \frac{d}{d t}
$$

the function $f: J \times \mathbb{R} \rightarrow \mathbb{R}, J=[0, T]=\{0\} \cup\left(\bigcup_{0}^{m} J_{k}\right), t_{m+1}=T$, the functions $\phi_{k}, \phi_{k}^{*}:$ $\mathbb{R} \rightarrow \mathbb{R}, k=1, \ldots, m$, are continuous, $\beta_{i}, \lambda_{i}, i=1,2$, are given constants, and $x\left(t_{k}^{+}\right)=$ $\lim _{\varepsilon \rightarrow 0+} x\left(t_{k}+\varepsilon\right), x\left(t_{k}^{-}\right)=x\left(t_{k}\right)$. For example, if $\alpha_{0}=\alpha_{1}=2, m=1$, and $g_{k}(t)=e^{(k+1) t}, k=0,1$, then (1.1) is reduced to

$$
\left\{\begin{array}{l}
e^{-2 t}\left(\frac{d^{2} x(t)}{d t^{2}}-\frac{d x(t)}{d t}\right)=f(t, x(t)), \quad t \in\left(t_{0}, t_{1}\right], \\
\frac{1}{4} e^{-4 t}\left(\frac{d^{2} x(t)}{d t^{2}}-2 \frac{d x(t)}{d t}\right)=f(t, x(t)), \quad t \in\left(t_{1}, T\right], \\
x\left(t_{1}^{+}\right)-x\left(t_{1}^{-}\right)=\phi_{1}\left(x\left(t_{1}\right)\right), \quad\left(\frac{1}{2} e^{-2 t} \frac{d x}{d t}\right)_{t=t_{1}^{+}}-\left(e^{-t} \frac{d x}{d t}\right)_{t=t_{1}^{-}}=\phi_{k}^{*}\left(x\left(t_{1}\right)\right), \\
x(0)+\beta_{1}\left(e^{-t} \frac{d x}{d t}\right)_{t=0}=\lambda_{1}, \quad x(T)+\beta_{2}\left(\frac{1}{2} e^{-2 t} \frac{d x}{d t}\right)_{t=T}=\lambda_{2},
\end{array}\right.
$$

with $D_{g_{k}}=\frac{e^{-(k+1) t}}{(k+1)} \frac{d}{d t}$ for $k=0,1$.

For $\gamma>0$, the Riemann-Liouville fractional integral of an integrable function $h:[a, b] \rightarrow$ $\mathbb{R}$ with respect to an increasing function $g \in C^{n}([a, b])$ such that $g^{\prime}(t) \neq 0$ for all $t \in[a, b]$ is defined by [9-11]

$$
{ }_{a} I_{g}^{\gamma} h(t)=\frac{1}{\Gamma(\gamma)} \int_{a}^{t} \frac{g^{\prime}(s) h(s)}{[g(t)-g(s)]^{1-\gamma}} d s,
$$

where $\Gamma$ is the gamma function. The Riemann-Liouville fractional derivative of a function $h$ with respect to another function $g$ on $[a, b]$ is defined as

$$
{ }_{a}^{\star} D_{g}^{\gamma} h(t)=D_{g a}^{n} I_{g}^{n-\gamma} h(t)=\frac{1}{\Gamma(n-\gamma)} D_{g}^{n} \int_{a}^{t} \frac{g^{\prime}(s) h(s)}{[g(t)-g(s)]^{1+\gamma-n}} d s,
$$

whereas the Caputo derivative is defined by

$$
{ }_{a} D_{g}^{\gamma} h(t)={ }_{a} I_{g}^{n-\gamma} D_{g}^{n} h(t)=\frac{1}{\Gamma(n-\gamma)} \int_{a}^{t} \frac{g^{\prime}(s) D_{g}^{n} h(s)}{[g(t)-g(s)]^{1+\gamma-n}} d s,
$$

where $D_{g}^{n}=\underbrace{D_{g} \cdots D_{g}}_{n \text { times }}, D_{g}$ is defined in (1.2), and $n$ is a positive integer such that $n-1<$ $\gamma<n$. There are relations of fractional integral and derivatives of the Riemann-Liouville and Caputo types, which will be used in our investigation [9]:

$$
{ }_{a} I_{g}^{\gamma}\left({ }_{a}^{\star} D_{g}^{\gamma} h\right)(t)=h(t)-\sum_{j=1}^{n} \frac{(g(t)-g(a))^{\gamma-j}}{\Gamma(\gamma-j+1)} D_{g}^{n-j}\left({ }_{a} I_{g}^{n-\gamma} h\right)(a)
$$

and

$$
{ }_{a} I_{g}^{\gamma}\left({ }_{a} D_{g}^{\gamma} h\right)(t)=h(t)-\sum_{j=0}^{n-1} \frac{(g(t)-g(a))^{j}}{j !} D_{g}^{j} h(a) .
$$


In addition, for $\gamma, \delta>0$, the relation

$$
{ }_{a} I_{g}^{\gamma}(g(t)-g(a))^{\delta}=\frac{\Gamma(\delta+1)}{\Gamma(\gamma+\delta+1)}(g(t)-g(a))^{\gamma+\delta},
$$

is applied in the main results [9]. For some recent results, we refer the interested the reader to [12-17].

Note that (1.4) is reduced to the Riemann-Liouville and Hadamard fractional integrals when $g(t)=t$ and $g(t)=\log t$, respectively, where $\log (\cdot)=\log _{e}(\cdot)$. The fractional derivatives of Hadamard and Hadamard-Caputo types can be obtained by substituting $g(t)=\log t$ into (1.5) and (1.6), respectively. Also, the Riemann-Liouville and Caputo fractional derivatives are presented by replacing $g(t)=t$ in (1.5) and (1.6), respectively. In the particular case for $g(t)=e^{t}$ of fractional differential equations and boundary value problems, the reader can find some results in $[18,19]$. Therefore problem $(1.1)$ generates many types and also mixed types of impulsive fractional differential equations with boundary conditions.

Using standard fixed point theorems, we study the existence and uniqueness of solutions for the impulsive fractional boundary value problem (1.1). An auxiliary result useful to transform problem (1.1) into an equivalent integral equation is proved in Sect. 2. The main results are presented in Sect. 3, where an existence and uniqueness result is proved via Banach's fixed point theorem and an existence result with the help of the Leray-Schauder nonlinear alternative. Some illustrative examples are constructed in Sect. 4.

\section{An auxiliary lemma}

In this section, we prove an auxiliary lemma, which is the basic tool to express the solution of problem (1.1) in an equivalent integral equation. Note that two boundary conditions in (1.1) are separated: one condition is stated at the initial point $t=0$, and the other at the end point $t=T$. We use the notation

$$
\left\langle t_{j+1}\right\rangle= \begin{cases}t, & t_{j+1}>t_{k}, \\ t_{j+1}, & t_{j+1} \leq t_{k} .\end{cases}
$$

For example, if $t \in\left(t_{3}, t_{4}\right]$, then $t_{k}=t_{3}$, and $\sum_{j=0}^{3}\left\langle t_{j+1}\right\rangle=\left\langle t_{1}\right\rangle+\left\langle t_{2}\right\rangle+\left\langle t_{3}\right\rangle+\left\langle t_{4}\right\rangle=t_{1}+t_{2}+t_{3}+t$. In addition, we define $G_{j}=g_{j}\left(\left\langle t_{j+1}\right\rangle\right)-g_{j}\left(t_{j}\right)$ and also the nonzero constant

$$
\Omega=\left(\beta_{2}-\beta_{1}\right)+\sum_{j=0}^{m} G_{j}
$$

The next lemma concerns a linear variant of problem (1.1).

Lemma 2.1 The separated boundary value problem of impulsive fractional differential equations with respect to other functions

$$
\left\{\begin{array}{l}
t_{k} D_{g_{k}}^{\alpha_{k}} x(t)=y(t), \quad t \neq t_{k}, k=0,1,2, \ldots, m, \\
x\left(t_{k}^{+}\right)-x\left(t_{k}^{-}\right)=\phi_{k}\left(x\left(t_{k}\right)\right), \quad k=1,2, \ldots, m, \\
D_{g_{k}} x\left(t_{k}^{+}\right)-D_{g_{k-1}} x\left(t_{k}^{-}\right)=\phi_{k}^{*}\left(x\left(t_{k}\right)\right), \quad k=1,2, \ldots, m, \\
x(0)+\beta_{1} D_{g_{0}} x(0)=\lambda_{1}, \quad x(T)+\beta_{2} D_{g_{m}} x(T)=\lambda_{2},
\end{array}\right.
$$


is equivalent to the integral equation

$$
\begin{aligned}
x(t)= & \frac{\lambda_{1}}{\Omega}\left(\beta_{2}+\left(\sum_{j=0}^{m} G_{j}\right)-\left(\sum_{j=0}^{k} G_{j}\right)\right) \\
& +\frac{1}{\Omega}\left(-\beta_{1}+\left(\sum_{j=0}^{k} G_{j}\right)\right)\left\{\lambda_{2}-\sum_{j=0}^{m-1}\left[\left(t_{j} I_{g_{j}}^{\alpha_{j}} y\right)\left(t_{j+1}\right)+\phi_{j+1}\left(x\left(t_{j+1}\right)\right)\right]\right. \\
& -\left(t_{m} I_{g_{m}}^{\alpha_{m}} y\right)(T)-\sum_{j=0}^{m-1} G_{j+1}\left(\sum_{r=0}^{j}\left[\left(t_{r} I_{g_{r}}^{\alpha_{r}-1} y\right)\left(t_{r+1}\right)+\phi_{r+1}^{*}\left(x\left(t_{r+1}\right)\right)\right]\right) \\
& \left.-\beta_{2}\left(t_{m} I_{g_{m}}^{\alpha_{m}-1} y\right)(T)-\beta_{2} \sum_{j=0}^{m-1}\left[\left({ }_{t_{j}} I_{g_{j}}^{\alpha_{j}-1} y\right)\left(t_{j+1}\right)+\phi_{j+1}^{*}\left(x\left(t_{j+1}\right)\right)\right]\right\} \\
& +\sum_{j=0}^{k-1}\left[\left({ }_{t j} I_{g_{j}}^{\alpha_{j}} y\right)\left(t_{j+1}\right)+\phi_{j+1}\left(x\left(t_{j+1}\right)\right)\right] \\
& +\sum_{j=0}^{k-1} G_{j+1}\left(\sum_{r=0}^{j}\left[\left(t_{r} I_{g_{r}}^{\alpha_{r}-1} y\right)\left(t_{r+1}\right)+\phi_{r+1}^{*}\left(x\left(t_{r+1}\right)\right)\right]\right) \\
& +\left(t_{k} I_{g_{k}}^{\alpha_{k}} y\right)(t), \quad t \in J_{k} .
\end{aligned}
$$

Proof First, we transform problem (1.1) into an integral equation by applying the fractional integral of order $1<\alpha_{0} \leq 2$ for $t \in\left(t_{0}, t_{1}\right]$ with respect to a function $g_{0}(t)$ to both sides of equation (1.1) and also using (1.8):

$$
\begin{aligned}
t_{0} I_{g_{0}}^{\alpha_{0}}\left(t_{0} D_{g_{0}}^{\alpha_{0}} x\right)(t) & =x(t)-c_{0}-c_{1}\left(g_{0}(t)-g_{0}\left(t_{0}\right)\right) \\
& =\left(t_{0} I_{g_{0}}^{\alpha_{0}} y\right)(t),
\end{aligned}
$$

where $c_{0}=x\left(t_{0}^{+}\right)$and $c_{1}=D_{g_{0}} x\left(t_{0}^{+}\right)$. After that, from (2.3) we obtain

$$
D_{g_{0}} x(t)=c_{1}+\left({ }_{t_{0}} I_{g_{0}}^{\alpha_{0}-1} y\right)(t)
$$

For $t \in\left(t_{1}, t_{2}\right]$, by applying the fractional integral of order $1<\alpha_{1} \leq 2$ with respect to the function $g_{1}(t)$ to the first equation of (1.1) and again using (1.8), we have

$$
x(t)=x\left(t_{1}^{+}\right)+\left(g_{1}(t)-g_{1}\left(t_{1}\right)\right) D_{g_{1}} x\left(t_{1}^{+}\right)+\left({ }_{t_{1}} I_{g_{1}}^{\alpha_{1}} y\right)(t)
$$

From the impulsive conditions $x\left(t_{1}^{+}\right)=x\left(t_{1}\right)+\phi_{1}\left(x\left(t_{1}\right)\right)$ and $D_{g_{1}} x\left(t_{1}^{+}\right)=D_{g_{0}} x\left(t_{1}\right)+\phi_{1}^{*}\left(x\left(t_{1}\right)\right)$ we have

$$
\begin{aligned}
x(t)= & c_{0}+c_{1}\left[\left(g_{0}\left(t_{1}\right)-g_{0}\left(t_{0}\right)\right)+\left(g_{1}(t)-g_{1}\left(t_{1}\right)\right)\right]+\left(t_{0} I_{g_{0}}^{\alpha_{0}} y\right)\left(t_{1}\right)+\phi_{1}\left(x\left(t_{1}\right)\right) \\
& +\left(g_{1}(t)-g_{1}\left(t_{1}\right)\right)\left[\left(t_{0} I_{g_{0}}^{\alpha_{0}-1} y\right)\left(t_{1}\right)+\phi_{1}^{*}\left(x\left(t_{1}\right)\right)\right]+\left({ }_{t_{1}} I_{g_{1}}^{\alpha_{1}} y\right)(t) .
\end{aligned}
$$

For $t \in\left(t_{2}, t_{3}\right]$, using the fractional integral of order $1<\alpha_{2} \leq 2$ with respect to the function $g_{2}(t)$ to both sides of the first equation in (1.1), we obtain

$$
x(t)=x\left(t_{2}^{+}\right)+\left(g_{2}(t)-g_{2}\left(t_{2}\right)\right) D_{g_{2}} x\left(t_{2}^{+}\right)+\left({ }_{t_{2}} I_{g_{2}}^{\alpha_{2}} y\right)(t) .
$$


By substituting impulsive conditions we obtain

$$
\begin{aligned}
x(t)= & c_{0}+c_{1}\left[\left(g_{0}\left(t_{1}\right)-g_{0}\left(t_{0}\right)\right)+\left(g_{1}\left(t_{2}\right)-g_{1}\left(t_{1}\right)\right)+\left(g_{2}(t)-g_{2}\left(t_{2}\right)\right)\right] \\
& +\left(t_{0} I_{g_{0}}^{\alpha_{0}} y\right)\left(t_{1}\right)+\phi_{1}\left(x\left(t_{1}\right)\right)+\left({ }_{t_{1}} I_{g_{1}}^{\alpha_{1}} y\right)\left(t_{2}\right)+\phi_{2}\left(x\left(t_{2}\right)\right) \\
& +\left(g_{1}\left(t_{2}\right)-g_{1}\left(t_{1}\right)\right)\left[\left(t_{0} I_{g_{0}}^{\alpha_{0}-1} y\right)\left(t_{1}\right)+\phi_{1}^{*}\left(x\left(t_{1}\right)\right)\right] \\
& +\left(g_{2}(t)-g_{2}\left(t_{2}\right)\right)\left[\left({ }_{t_{0}} I_{g_{0}}^{\alpha_{0}-1} y\right)\left(t_{1}\right)+\phi_{1}^{*}\left(x\left(t_{1}\right)\right)+\left({ }_{t_{1}} I_{g_{1}}^{\alpha_{1}-1} y\right)\left(t_{2}\right)+\phi_{2}^{*}\left(x\left(t_{2}\right)\right)\right] \\
& +\left({ }_{t_{2}} I_{g_{2}}^{\alpha_{2}} y\right)(t) .
\end{aligned}
$$

Repeating the procedure, for any $t \in\left(t_{k}, t_{k+1}\right], k=0,1,2, \ldots, m$, we get the integral equation

$$
\begin{aligned}
x(t)= & c_{0}+c_{1} \sum_{j=0}^{k}\left[g_{j}\left(\left\langle t_{j+1}\right\rangle\right)-g_{j}\left(t_{j}\right)\right]+\sum_{j=0}^{k-1}\left[\left({ }_{t_{j}} I_{g_{j}}^{\alpha_{j}} y\right)\left(t_{j+1}\right)+\phi_{j+1}\left(x\left(t_{j+1}\right)\right)\right] \\
& +\sum_{j=0}^{k-1}\left[g_{j+1}\left(\left\langle t_{j+2}\right\rangle\right)-g_{j+1}\left(t_{j+1}\right)\right]\left(\sum_{r=0}^{j}\left[\left(t_{r} I_{g_{r}}^{\alpha_{r}-1} y\right)\left(t_{r+1}\right)+\phi_{r+1}^{*}\left(x\left(t_{r+1}\right)\right)\right]\right) \\
& +\left(t_{k} I_{g_{k}}^{\alpha_{k}} y\right)(t) .
\end{aligned}
$$

From the first boundary condition in (1.1) we have

$$
c_{0}+\beta_{1} c_{1}=\lambda_{1}
$$

Since

$$
\begin{aligned}
x(T)= & c_{0}+c_{1} \sum_{j=0}^{m}\left[g_{j}\left(t_{j+1}\right)-g_{j}\left(t_{j}\right)\right]+\sum_{j=0}^{m-1}\left[\left(t_{j} I_{g_{j}}^{\alpha_{j}} y\right)\left(t_{j+1}\right)+\phi_{j+1}\left(x\left(t_{j+1}\right)\right)\right] \\
& +\sum_{j=0}^{m-1}\left[g_{j+1}\left(t_{j+2}\right)-g_{j+1}\left(t_{j+1}\right)\right]\left(\sum_{r=0}^{j}\left[\left(t_{r} I_{g_{r}}^{\alpha_{r}-1} y\right)\left(t_{r+1}\right)+\phi_{r+1}^{*}\left(x\left(t_{r+1}\right)\right)\right]\right) \\
& +\left(t_{m} I_{g_{m}}^{\alpha_{m}} y\right)(T)
\end{aligned}
$$

and

$$
D_{g_{m}} x(T)=c_{1}+\sum_{j=0}^{m-1}\left[\left(t_{j} I_{g_{j}}^{\alpha_{j}-1} y\right)\left(t_{j+1}\right)+\phi_{j+1}^{*}\left(x\left(t_{j+1}\right)\right)\right]+\left(t_{m} I_{g_{m}}^{\alpha_{m}-1} y\right)(T),
$$

by substituting in the second boundary condition of (1.1) we obtain

$$
\begin{aligned}
c_{0} & +c_{1}\left(\beta_{2}+\sum_{j=0}^{m}\left[g_{j}\left(t_{j+1}\right)-g_{j}\left(t_{j}\right)\right]\right) \\
& =\lambda_{2}-\sum_{j=0}^{m-1}\left[\left({ }_{t j} I_{g_{j}}^{\alpha_{j}} y\right)\left(t_{j+1}\right)+\phi_{j+1}\left(x\left(t_{j+1}\right)\right)\right]-\left({ }_{t_{m}} I_{g_{m}}^{\alpha_{m}} y\right)(T)-\beta_{2}\left(t_{m} I_{g_{m}}^{\alpha_{m}-1} y\right)(T)
\end{aligned}
$$




$$
\begin{aligned}
& -\sum_{j=0}^{m-1}\left[g_{j+1}\left(t_{j+2}\right)-g_{j+1}\left(t_{j+1}\right)\right]\left(\sum_{r=0}^{j}\left[\left(t_{r} I_{g_{r}}^{\alpha_{r}-1} y\right)\left(t_{r+1}\right)+\phi_{r+1}^{*}\left(x\left(t_{r+1}\right)\right)\right]\right) \\
& -\beta_{2} \sum_{j=0}^{m-1}\left[\left({ }_{t j} I_{g_{j}}^{\alpha_{j}-1} y\right)\left(t_{j+1}\right)+\phi_{j+1}^{*}\left(x\left(t_{j+1}\right)\right)\right] .
\end{aligned}
$$

Solving the two linear equations (2.5) and (2.6) for constants $c_{0}$ and $c_{1}$, we obtain

$$
\begin{aligned}
c_{0}= & \frac{\lambda_{1}}{\Omega}\left(\beta_{2}+\sum_{j=0}^{m} G_{j}\right)-\frac{\beta_{1}}{\Omega}\left\{\lambda_{2}-\sum_{j=0}^{m-1}\left[\left(t_{j} I_{g_{j}}^{\alpha_{j}} y\right)\left(t_{j+1}\right)+\phi_{j+1}\left(x\left(t_{j+1}\right)\right)\right]\right. \\
& -\left(t_{m} I_{g_{m}}^{\alpha_{m}} y\right)(T)-\sum_{j=0}^{m-1} G_{j+1}\left(\sum_{r=0}^{j}\left[\left(t_{r_{r}} g_{g_{r}}^{\alpha_{r}-1} y\right)\left(t_{r+1}\right)+\phi_{r+1}^{*}\left(x\left(t_{r+1}\right)\right)\right]\right) \\
& \left.-\beta_{2}\left(t_{m} I_{g_{m}}^{\alpha_{m}-1} y\right)(T)-\beta_{2} \sum_{j=0}^{m-1}\left[\left({ }_{t_{j}} I_{g_{j}}^{\alpha_{j}-1} y\right)\left(t_{j+1}\right)+\phi_{j+1}^{*}\left(x\left(t_{j+1}\right)\right)\right]\right\}
\end{aligned}
$$

and

$$
\begin{aligned}
c_{1}= & \frac{1}{\Omega}\left\{\left(\lambda_{2}-\lambda_{1}\right)-\sum_{j=0}^{m-1}\left[\left({ }_{t_{j}} I_{g_{j}}^{\alpha_{j}} y\right)\left(t_{j+1}\right)+\phi_{j+1}\left(x\left(t_{j+1}\right)\right)\right]-\left(t_{m} I_{g_{m}}^{\alpha_{m}} y\right)(T)\right. \\
& -\sum_{j=0}^{m-1} G_{j+1}\left(\sum_{r=0}^{j}\left[\left(t_{r} I_{g_{r}}^{\alpha_{r}-1} y\right)\left(t_{r+1}\right)+\phi_{r+1}^{*}\left(x\left(t_{r+1}\right)\right)\right]\right) \\
& \left.-\beta_{2}\left(t_{m} I_{g_{m}}^{\alpha_{m}-1} y\right)(T)-\beta_{2} \sum_{j=0}^{m-1}\left[\left(t_{j} I_{g_{j}}^{\alpha_{j}-1} y\right)\left(t_{j+1}\right)+\phi_{j+1}^{*}\left(x\left(t_{j+1}\right)\right)\right]\right\} .
\end{aligned}
$$

Substituting the values of $c_{0}$ and $c_{1}$ into (2.4), we obtain (2.2). The converse follows by direct computation. The proof is completed.

Remark 2.2 If $k=0$, then problem (2.1) is reduced to a nonimpulsively fractional differential equation with boundary conditions of the form

$$
\left\{\begin{array}{l}
t_{0} D_{g_{0}}^{\alpha_{0}} x(t)=y(t), \quad t \in(0, T), \\
x(0)+\beta_{1} D_{g_{0}} x(0)=\lambda_{1}, \quad x(T)+\beta_{2} D_{g_{0}} x(T)=\lambda_{2} .
\end{array}\right.
$$

Consequently, the result in Theorem 2.1 can be presented by

$$
\begin{aligned}
x(t)= & \frac{\lambda_{1}}{\Omega}\left(\beta_{2}+g_{0}(T)-g_{0}(t)\right)+\frac{1}{\Omega}\left(-\beta_{1}+g_{0}(t)-g_{0}\left(t_{0}\right)\right)\left\{\lambda_{2}-\left({ }_{t_{0}} I_{g_{0}}^{\alpha_{0}} y\right)(T)\right. \\
& \left.-\beta_{2}\left(t_{0} I_{g_{0}}^{\alpha_{0}-1} y\right)(T)\right\}+\left({ }_{t_{0}} I_{g_{0}}^{\alpha_{0}} y\right)(t), \quad t \in[0, T],
\end{aligned}
$$

where $\Omega=\left(\beta_{2}-\beta_{1}\right)+\left(g_{0}(T)-g_{0}\left(t_{0}\right)\right)$, provided that $\Omega \neq 0$.

\section{Main results}

In the section, we establish the existence and uniqueness results for problem (1.1). We define the space of piecewise continuous functions $\operatorname{PC}(J, \mathbb{R})=\{x: J \rightarrow \mathbb{R}: x(t)$ is continuous everywhere except for some $t_{k}$ at which $x\left(t_{k}^{+}\right)$and $x\left(t_{k}^{-}\right)$exist and $x\left(t_{k}^{-}\right)=x\left(t_{k}\right)$, 
$k=1,2, \ldots, m\}$. Note that $\operatorname{PC}(J, \mathbb{R})$ is a Banach space equipped with the norm $\|x\|=$ $\sup \{|x(t)|: t \in J\}$.

In the following, for the convenience of the reader, we express the fractional integral defined in (1.4) of a nonlinear two-variable function $f(t, x(t))$ by a subscript notation by

$$
\left({ }_{a} I_{g}^{\gamma} f_{x}\right)(t):={ }_{a} I_{g}^{\gamma} f(t, x(t))=\frac{1}{\Gamma(\gamma)} \int_{a}^{t} \frac{g^{\prime}(s) f(s, x(s))}{[g(t)-g(s)]^{1-\gamma}} d s
$$

In view of Lemma 2.1, to establish existence theorems, we consider the operator equation $x=\mathcal{A} x$, where $\mathcal{A}: \mathrm{PC}(J, \mathbb{R}) \rightarrow \mathrm{PC}(J, \mathbb{R})$ is defined by

$$
\begin{aligned}
\mathcal{A} x(t)= & \frac{\lambda_{1}}{\Omega}\left(\beta_{2}+\left(\sum_{j=0}^{m} G_{j}\right)-\left(\sum_{j=0}^{k} G_{j}\right)\right) \\
& +\frac{1}{\Omega}\left(-\beta_{1}+\left(\sum_{j=0}^{k} G_{j}\right)\right)\left\{\lambda_{2}-\sum_{j=0}^{m-1}\left[\left(t_{j} I_{g_{j}}^{\alpha_{j}} f_{x}\right)\left(t_{j+1}\right)+\phi_{j+1}\left(x\left(t_{j+1}\right)\right)\right]\right. \\
& -\left(t_{m} I_{g_{m}}^{\alpha_{m}} f_{x}\right)(T)-\sum_{j=0}^{m-1} G_{j+1}\left(\sum_{r=0}^{j}\left[\left(t_{r} I_{g_{r}}^{\alpha_{r}-1} f_{x}\right)\left(t_{r+1}\right)+\phi_{r+1}^{*}\left(x\left(t_{r+1}\right)\right)\right]\right) \\
& \left.-\beta_{2}\left(t_{m} I_{g_{m}}^{\alpha_{m}-1} f_{x}\right)(T)-\beta_{2} \sum_{j=0}^{m-1}\left[\left({ }_{t_{j}} I_{g_{j}}^{\alpha_{j}-1} f_{x}\right)\left(t_{j+1}\right)+\phi_{j+1}^{*}\left(x\left(t_{j+1}\right)\right)\right]\right\} \\
& +\sum_{j=0}^{k-1}\left[\left(t_{j} I_{g_{j}}^{\alpha_{j}} f_{x}\right)\left(t_{j+1}\right)+\phi_{j+1}\left(x\left(t_{j+1}\right)\right)\right] \\
& +\sum_{j=0}^{k-1} G_{j+1}\left(\sum_{r=0}^{j}\left[\left(t_{r} I_{g_{r}}^{\alpha_{r}-1} f_{x}\right)\left(t_{r+1}\right)+\phi_{r+1}^{*}\left(x\left(t_{r+1}\right)\right)\right]\right) \\
& +\left(t_{k} I_{g_{k}}^{\alpha_{k}} f_{x}\right)(t), \quad t \in J_{k},
\end{aligned}
$$

and also apply fixed point theory.

Next, we use the Lipschitz condition of a nonlinear function $f$ to prove the existence of a unique solution using the Banach fixed point theorem.

For convenience, we denote

$$
\begin{aligned}
Q_{1}= & \frac{\left|\lambda_{1}\right|}{|\Omega|}\left(\left|\beta_{2}\right|+2 \sum_{j=0}^{m}\left|G_{j}\right|\right), \quad Q_{2}=\frac{1}{|\Omega|}\left(\left|\beta_{1}\right|+\sum_{j=0}^{m}\left|G_{j}\right|\right), \\
Q_{3}= & \left(Q_{2}+1\right) \sum_{j=0}^{m} \frac{\left(g_{j}\left(t_{j+1}\right)-g_{j}\left(t_{j}\right)\right)^{\alpha_{j}}}{\Gamma\left(\alpha_{j}+1\right)}+Q_{2}\left|\beta_{2}\right| \sum_{j=0}^{m} \frac{\left(g_{j}\left(t_{j+1}\right)-g_{j}\left(t_{j}\right)\right)^{\alpha_{j}-1}}{\Gamma\left(\alpha_{j}\right)} \\
& +\left(Q_{2}+1\right) \sum_{j=0}^{m-1}\left|G_{j+1}\right| \sum_{r=0}^{j} \frac{\left(g_{r}\left(t_{r+1}\right)-g_{r}\left(t_{r}\right)\right)^{\alpha_{r}-1}}{\Gamma\left(\alpha_{r}\right)}, \\
Q_{4}= & Q_{2}\left|\beta_{2}\right| m+\left(Q_{2}+1\right) \sum_{j=0}^{m-1}\left|G_{j+1}\right|(j+1),
\end{aligned}
$$


and

$$
Q_{5}=\left(Q_{2}+1\right) m
$$

Theorem 3.1 Let $g_{k} \in C^{2}([0, T])$ with $g_{k}^{\prime}(t)>0$ for $t \in[0, T], k=0,1, \ldots, m$. Assume that functions $f: J \times \mathbb{R} \rightarrow \mathbb{R}, \phi_{k}: \mathbb{R} \rightarrow \mathbb{R}$, and $\phi_{k}^{*}: \mathbb{R} \rightarrow \mathbb{R}, k=1,2, \ldots, m$, satisfy

$\left(H_{1}\right)|f(t, x)-f(t, y)| \leq L_{1}|x-y|, L_{1}>0, \forall t \in J, x, y \in \mathbb{R}$,

$\left(H_{2}\right)\left|\phi_{k}(x)-\phi_{k}(y)\right| \leq L_{2}|x-y|,\left|\phi_{k}^{*}(x)-\phi_{k}^{*}(y)\right| \leq L_{3}|x-y|, L_{2}, L_{3}>0, \forall x, y \in \mathbb{R}$.

If

$$
L_{1} Q_{3}+L_{2} Q_{5}+L_{3} Q_{4}<1
$$

then the separated boundary value problem of impulsive fractional differential equations with respect to other functions (1.1) has a unique solution on J.

Proof The Banach fixed point theorem guarantees a unique solution in a set $B_{\rho}=$ $\{x \in \mathrm{PC}(J, \mathbb{R}):\|x\| \leq \rho\}$, where $\rho$ is chosen such that

$$
\rho>\frac{Q_{1}+\left|\lambda_{2}\right| Q_{2}+\rho\left(L_{1} Q_{3}+L_{2} Q_{5}+L_{3} Q_{4}\right)+M_{1} Q_{3}+M_{2} Q_{5}+M_{3} Q_{4}}{1-\left(L_{1} Q_{3}+L_{2} Q_{5}+L_{3} Q_{4}\right)} .
$$

We set $\sup _{t \in J}\left|f_{0}\right|=M_{1}, \max _{k}\left|\phi_{k}(0)\right|=M_{2}$, and $\max _{k}\left|\phi_{k}^{*}(0)\right|=M_{3}$, where $f_{0}=f(t, 0)$. Using $\left|f_{x}\right| \leq\left|f_{x}-f_{0}\right|+\left|f_{0}\right|,\left|\phi_{k}(x)\right| \leq\left|\phi_{k}(x)-\phi_{k}(0)\right|+\left|\phi_{k}(0)\right|$, and $\left|\phi_{k}^{*}(x)\right| \leq\left|\phi_{k}^{*}(x)-\phi_{k}^{*}(0)\right|+\left|\phi_{k}^{*}(0)\right|$, $k=1,2,3, \ldots, m$, for any $x \in B_{r}$, we have

$$
\begin{aligned}
& |\mathcal{A} x(t)| \\
& \leq \frac{\left|\lambda_{1}\right|}{|\Omega|}\left(\left|\beta_{2}\right|+\left(\sum_{j=0}^{m}\left|G_{j}\right|\right)+\left(\sum_{j=0}^{k}\left|G_{j}\right|\right)\right) \\
& +\frac{1}{|\Omega|}\left(\left|\beta_{1}\right|+\left(\sum_{j=0}^{k}\left|G_{j}\right|\right)\right)\left\{\left|\lambda_{2}\right|+\sum_{j=0}^{m-1}\left[\left({ }_{t_{j}} I_{g_{j}}^{\alpha_{j}}\left|f_{x}\right|\right)\left(t_{j+1}\right)+\left|\phi_{j+1}\left(x\left(t_{j+1}\right)\right)\right|\right]\right. \\
& +\left({ }_{t_{m}} I_{g_{m}}^{\alpha_{m}}\left|f_{x}\right|\right)(T)+\sum_{j=0}^{m-1}\left|G_{j+1}\right|\left(\sum_{r=0}^{j}\left[\left({ }_{t_{r}} I_{g_{r}}^{\alpha_{r}-1}\left|f_{x}\right|\right)\left(t_{r+1}\right)+\left|\phi_{r+1}^{*}\left(x\left(t_{r+1}\right)\right)\right|\right]\right) \\
& \left.+\left|\beta_{2}\right|\left(t_{m} I_{g_{m}}^{\alpha_{m}-1}\left|f_{x}\right|\right)(T)+\left|\beta_{2}\right| \sum_{j=0}^{m-1}\left[\left({ }_{t_{j}} I_{g_{j}}^{\alpha_{j}-1}\left|f_{x}\right|\right)\left(t_{j+1}\right)+\left|\phi_{j+1}^{*}\left(x\left(t_{j+1}\right)\right)\right|\right]\right\} \\
& \left.+\sum_{j=0}^{k-1}\left[\left(t_{j} I_{g_{j}}^{\alpha_{j}}\left|f_{x}\right|\right)\left(t_{j+1}\right)+\left|\phi_{j+1}\left(x\left(t_{j+1}\right)\right)\right|\right]+{ }_{t_{k}} I_{g_{k}}^{\alpha_{k}}\left|f_{x}\right|\right)(t) \\
& +\sum_{j=0}^{k-1}\left|G_{j+1}\right|\left(\sum_{r=0}^{j}\left[\left(t_{r} I_{g_{r}}^{\alpha_{r}-1}\left|f_{x}\right|\right)\left(t_{r+1}\right)+\left|\phi_{r+1}^{*}\left(x\left(t_{r+1}\right)\right)\right|\right]\right) \\
& \leq Q_{1}+\left|\lambda_{2}\right| Q_{2}+\rho\left(L_{1} Q_{3}+L_{2} Q_{5}+L_{3} Q_{4}\right)+M_{1} Q_{3}+M_{2} Q_{5}+M_{3} Q_{4} \\
& <\rho \text {. }
\end{aligned}
$$


This means the $\mathcal{A} B_{\rho} \subset B_{\rho}$. To prove the contractivity of the operator $\mathcal{A}$, for any $x, y \in B_{\rho}$, we consider

$$
\begin{aligned}
\mid \mathcal{A} x( & t)-\mathcal{A} y(t) \mid \\
\leq & \frac{1}{|\Omega|}\left(\left|\beta_{1}\right|+\left(\sum_{j=0}^{k}\left|G_{j}\right|\right)\right)\left\{\sum_{j=0}^{m-1}\left[\left(t_{j} I_{g_{j}}^{\alpha_{j}}\left|f_{x}-f_{y}\right|\right)\left(t_{j+1}\right)+\left|\phi_{j+1}\left(x\left(t_{j+1}\right)\right)-\phi_{j+1}\left(y\left(t_{j+1}\right)\right)\right|\right]\right. \\
& +\left(t_{m} I_{g_{m}}^{\alpha_{m}}\left|f_{x}-f_{y}\right|\right)(T)+\left|\beta_{2}\right|\left(t_{m} I_{g_{m}}^{\alpha_{m}-1}\left|f_{x}-f_{y}\right|\right)(T) \\
& +\sum_{j=0}^{m-1}\left|G_{j+1}\right|\left(\sum_{r=0}^{j}\left[\left(t_{r} I_{g_{r}}^{\alpha_{r}-1}\left|f_{x}-f_{y}\right|\right)\left(t_{r+1}\right)+\left|\phi_{r+1}^{*}\left(x\left(t_{r+1}\right)\right)-\phi_{r+1}^{*}\left(y\left(t_{r+1}\right)\right)\right|\right]\right) \\
& \left.+\left|\beta_{2}\right| \sum_{j=0}^{m-1}\left[\left(t_{j} I_{g_{j}}^{\alpha_{j}-1}\left|f_{x}-f_{y}\right|\right)\left(t_{j+1}\right)+\left|\phi_{j+1}^{*}\left(x\left(t_{j+1}\right)\right)-\phi_{j+1}^{*}\left(y\left(t_{j+1}\right)\right)\right|\right]\right\} \\
& +\sum_{j=0}^{k-1}\left[\left(t_{j} I_{g_{j}}^{\alpha_{j}}\left|f_{x}-f_{y}\right|\right)\left(t_{j+1}\right)+\left|\phi_{j+1}\left(x\left(t_{j+1}\right)\right)-\phi_{j+1}\left(y\left(t_{j+1}\right)\right)\right|\right] \\
& +\sum_{j=0}^{k-1}\left|G_{j+1}\right|\left(\sum_{r=0}^{j}\left[\left(t_{r} I_{g_{r}}^{\alpha_{r}-1}\left|f_{x}-f_{y}\right|\right)\left(t_{r+1}\right)+\left|\phi_{r+1}^{*}\left(x\left(t_{r+1}\right)\right)-\phi_{r+1}^{*}\left(y\left(t_{r+1}\right)\right)\right|\right]\right) \\
& +\left(t_{k} I_{g_{k}}^{\alpha_{k}}\left|f_{x}-f_{y}\right|\right)(t) \\
\leq & \left(L_{1} Q_{3}+L_{2} Q_{5}+L_{3} Q_{4}\right)\|x-y\|,
\end{aligned}
$$

which implies $\|\mathcal{A} x-\mathcal{A} y\| \leq\left(L_{1} Q_{3}+L_{2} Q_{5}+L_{3} Q_{4}\right)\|x-y\|$. Since inequality (3.1) holds, we get that the operator $\mathcal{A}$ is a contraction. The benefit of the Banach contraction principle leads to the existence of a unique fixed point of operator $\mathcal{A}$, which is a unique solution of problem (1.1) on $J$. The proof is finished.

If $m=0$, then $Q_{4}$ and $Q_{5}$ in the previous theorem are reduced to zero, and the constants $Q_{1}, Q_{2}, Q_{3}$ are reduced to

$$
\begin{aligned}
& Q_{1}^{*}=\frac{\left|\lambda_{1}\right|}{|\Omega|}\left(\left|\beta_{2}\right|+2\left|g_{0}(T)-g_{0}(0)\right|\right), \\
& Q_{2}^{*}=\frac{1}{|\Omega|}\left(\left|\beta_{1}\right|+\left|g_{0}(T)-g_{0}(0)\right|\right), \\
& Q_{3}^{*}=\left(Q_{2}^{*}+1\right) \frac{\left(g_{0}(T)-g_{0}(0)\right)^{\alpha_{0}}}{\Gamma\left(\alpha_{0}+1\right)}+Q_{2}^{*}\left|\beta_{2}\right| \frac{\left(g_{0}(T)-g_{0}(0)\right)^{\alpha_{0}-1}}{\Gamma\left(\alpha_{0}\right)},
\end{aligned}
$$

respectively. Consequently, we get a result for nonimpulse effects.

Corollary 3.2 Assume that the function $f$ satisfies $\left(H_{1}\right)$. If $Q_{3}^{*} L_{1}<1$, then there exists a unique solution on $[0, T]$ of the problem

$$
\left\{\begin{array}{l}
{ }_{t_{0}} D_{g_{0}}^{\alpha_{0}} x(t)=f(t, x(t)), \quad t \in(0, T), \\
x(0)+\beta_{1} D_{g_{0}} x(0)=\lambda_{1}, \quad x(T)+\beta_{2} D_{g_{0}} x(T)=\lambda_{2} .
\end{array}\right.
$$

The next existence result is based on the Leray-Schauder nonlinear alternative. 
Lemma 3.3 (Nonlinear alternative for single-valued maps [20]) Let E be a Banach space, let $C$ be a closed convex subset of $E$, and let $U$ be an open subset of $C$ such that $0 \in U$. Suppose that $F: \bar{U} \rightarrow C$ is a continuous and compact map, that is, $F(\bar{U})$ is a relatively compact subset of $C$. Then either

(i) F has a fixed point in $\bar{U}$, or

(ii) there is $u \in \partial U$ (the boundary of $U$ in $C$ ) and $\lambda \in(0,1)$ with $u=\lambda F(u$ ).

Theorem 3.4 Suppose that:

$\left(H_{3}\right)$ There exist a continuous nondecreasing function $\psi_{1}:[0, \infty) \rightarrow(0, \infty)$ and a continuous function $p: J \rightarrow \mathbb{R}^{+}$such that

$$
|f(t, x)| \leq p(t) \psi_{1}(|x|), \quad(t, x) \in J \times \mathbb{R}
$$

$\left(H_{4}\right)$ There exist two continuous nondecreasing functions $\psi_{2}, \psi_{3}:[0, \infty) \rightarrow(0, \infty)$ such that

$$
\left|\phi_{k}(x)\right| \leq \psi_{2}(|x|) \text { and }\left|\phi_{k}^{*}(x)\right| \leq \psi_{3}(|x|)
$$

for all $x \in \mathbb{R}, k=1,2, \ldots, m$.

$\left(H_{5}\right)$ There exists a constant $M>0$ such that

$$
\frac{M}{\left(Q_{1}+\left|\lambda_{2}\right| Q_{2}\right)+\left(\|p\| \psi_{1}(M) Q_{3}+\psi_{2}(M) Q_{5}+\psi_{3}(M) Q_{4}\right)}>1 .
$$

Then the impulsive fractional boundary value problem (1.1) has at least one solution on J.

Proof First, we prove the continuity of $\mathcal{A}$. Let $B_{\rho^{*}}=\left\{x \in \mathrm{PC}(J, \mathbb{R}):\|x\| \leq \rho^{*}, \rho^{*}>0\right\}$ be a bounded ball in $\operatorname{PC}(J, \mathbb{R})$, and let $\left\{x_{n}\right\}$ be a sequence such that $x_{n} \rightarrow x$ in $B_{\rho^{*}}$. From the continuity of $f$ on $J \times \mathbb{R}$ we obtain

$$
f\left(t, x_{n}(t)\right) \rightarrow f(t, x(t)) \quad \text { as } n \rightarrow \infty .
$$

Then, for any $t \in J$, we get

$$
\begin{aligned}
& \left|\mathcal{A} x_{n}(t)-\mathcal{A} x(t)\right| \\
& \leq \frac{1}{|\Omega|}\left(\left|\beta_{1}\right|+\left(\sum_{j=0}^{k}\left|G_{j}\right|\right)\right)\left\{\sum _ { j = 0 } ^ { m - 1 } \left[\left(_{t_{j}} I_{g_{j}}^{\alpha_{j}}\left|f_{x_{n}}-f_{x}\right|\right)\left(t_{j+1}\right)+\mid \phi_{j+1}\left(x_{n}\left(t_{j+1}\right)\right)\right.\right. \\
& \left.\quad-\phi_{j+1}\left(x\left(t_{j+1}\right)\right) \mid\right]+\left(t_{m} I_{g_{m}}^{\alpha_{m}}\left|f_{x_{n}}-f_{x}\right|\right)(T)+\left|\beta_{2}\right|\left(t_{m} I_{g_{m}}^{\alpha_{m}-1}\left|f_{x_{n}}-f_{x}\right|\right)(T) \\
& \quad+\sum_{j=0}^{m-1}\left|G_{j+1}\right|\left(\sum_{r=0}^{j}\left[\left({ }_{t r} I_{g_{r}}^{\alpha_{r}-1}\left|f_{x_{n}}-f_{x}\right|\right)\left(t_{r+1}\right)+\left|\phi_{r+1}^{*}\left(x_{n}\left(t_{r+1}\right)\right)-\phi_{r+1}^{*}\left(x\left(t_{r+1}\right)\right)\right|\right]\right) \\
& \left.\quad+\left|\beta_{2}\right| \sum_{j=0}^{m-1}\left[\left(t_{t_{j}} I_{g_{j}}^{\alpha_{j}-1}\left|f_{x_{n}}-f_{x}\right|\right)\left(t_{j+1}\right)+\left|\phi_{j+1}^{*}\left(x_{n}\left(t_{j+1}\right)\right)-\phi_{j+1}^{*}\left(x\left(t_{j+1}\right)\right)\right|\right]\right\} \\
& \quad+\sum_{j=0}^{k-1}\left[\left(t_{j} I_{g_{j}}^{\alpha_{j}}\left|f_{x_{n}}-f_{x}\right|\right)\left(t_{j+1}\right)+\left|\phi_{j+1}\left(x_{n}\left(t_{j+1}\right)\right)-\phi_{j+1}\left(x\left(t_{j+1}\right)\right)\right|\right]
\end{aligned}
$$




$$
\begin{aligned}
& +\sum_{j=0}^{k-1}\left|G_{j+1}\right|\left(\sum_{r=0}^{j}\left[\left(t_{r} I_{g_{r}}^{\alpha_{r}-1}\left|f_{x_{n}}-f_{x}\right|\right)\left(t_{r+1}\right)+\left|\phi_{r+1}^{*}\left(x_{n}\left(t_{r+1}\right)\right)-\phi_{r+1}^{*}\left(x\left(t_{r+1}\right)\right)\right|\right]\right) \\
& +\left(t_{k} I_{g_{k}}^{\alpha_{k}}\left|f_{x_{n}}-f_{x}\right|\right)(t),
\end{aligned}
$$

which leads to $\left\|\mathcal{A} x_{n}-\mathcal{A} x\right\| \rightarrow 0$ as $n \rightarrow \infty$. Therefore $\mathcal{A}$ is continuous.

Next, we prove that $\mathcal{A}$ maps bounded sets into bounded sets in $\operatorname{PC}(J, \mathbb{R})$. For any $x \in B_{\rho^{*}}$ and $t \in J$, we get

$$
\begin{aligned}
& |\mathcal{A} x(t)| \\
& \leq \frac{\left|\lambda_{1}\right|}{|\Omega|}\left(\left|\beta_{2}\right|+\left(\sum_{j=0}^{m}\left|G_{j}\right|\right)+\left(\sum_{j=0}^{k}\left|G_{j}\right|\right)\right) \\
& +\frac{1}{|\Omega|}\left(\left|\beta_{1}\right|+\left(\sum_{j=0}^{k}\left|G_{j}\right|\right)\right)\left\{\left|\lambda_{2}\right|+\sum_{j=0}^{m-1}\left[\left(t_{j} I_{g_{j}}^{\alpha_{j}}\left|f_{x}\right|\right)\left(t_{j+1}\right)+\left|\phi_{j+1}\left(x\left(t_{j+1}\right)\right)\right|\right]\right. \\
& +\left({ }_{t_{m}} I_{g_{m}}^{\alpha_{m}}\left|f_{x}\right|\right)(T)+\sum_{j=0}^{m-1}\left|G_{j+1}\right|\left(\sum_{r=0}^{j}\left[\left({ }_{t_{r}} I_{g_{r}}^{\alpha_{r}-1}\left|f_{x}\right|\right)\left(t_{r+1}\right)+\left|\phi_{r+1}^{*}\left(x\left(t_{r+1}\right)\right)\right|\right]\right) \\
& \left.+\left|\beta_{2}\right|\left(t_{m} I_{g_{m}}^{\alpha_{m}-1}\left|f_{x}\right|\right)(T)+\left|\beta_{2}\right| \sum_{j=0}^{m-1}\left[\left({ }_{t j} I_{g_{j}}^{\alpha_{j}-1}\left|f_{x}\right|\right)\left(t_{j+1}\right)+\left|\phi_{j+1}^{*}\left(x\left(t_{j+1}\right)\right)\right|\right]\right\} \\
& +\sum_{j=0}^{k-1}\left[\left({ }_{t j} I_{g_{j}}^{\alpha_{j}}\left|f_{x}\right|\right)\left(t_{j+1}\right)+\left|\phi_{j+1}\left(x\left(t_{j+1}\right)\right)\right|\right]+\left({ }_{t_{k}} I_{g_{k}}^{\alpha_{k}}\left|f_{x}\right|\right)(t) \\
& +\sum_{j=0}^{k-1}\left|G_{j+1}\right|\left(\sum_{r=0}^{j}\left[\left(t_{r} I_{g_{r}}^{\alpha_{r}-1}\left|f_{x}\right|\right)\left(t_{r+1}\right)+\left|\phi_{r+1}^{*}\left(x\left(t_{r+1}\right)\right)\right|\right]\right) \\
& \leq \frac{\left|\lambda_{1}\right|}{|\Omega|}\left(\left|\beta_{2}\right|+2 \sum_{j=0}^{m}\left|G_{j}\right|\right)+\frac{1}{|\Omega|}\left(\left|\beta_{1}\right|+\sum_{j=0}^{m}\left|G_{j}\right|\right) \\
& \times\left\{\left|\lambda_{2}\right|+\sum_{j=0}^{m-1}\left[\|p\| \psi_{1}(\|x\|)\left({ }_{t_{j}} I_{g_{j}}^{\alpha_{j}}(1)\right)\left(t_{j+1}\right)+\psi_{2}(\|x\|)\right]\right. \\
& +\|p\| \psi_{1}(\|x\|)\left(t_{t_{m}} I_{g_{m}}^{\alpha_{m}}(1)\right)(T) \\
& +\sum_{j=0}^{m-1}\left|G_{j+1}\right|\left(\sum_{r=0}^{j}\left[\|p\| \psi_{1}(\|x\|)\left({ }_{t_{r}} I_{g_{r}}^{\alpha_{r}-1}(1)\right)\left(t_{r+1}\right)+\psi_{3}(\|x\|)\right]\right) \\
& +\left|\beta_{2}\right|\|p\| \psi_{1}(\|x\|)\left(t_{m} I_{g_{m}}^{\alpha_{m}-1}(1)\right)(T) \\
& \left.+\left|\beta_{2}\right| \sum_{j=0}^{m-1}\left[\|p\| \psi_{1}(\|x\|)\left(t_{t_{j}} I_{g_{j}}^{\alpha_{j}-1}(1)\right)\left(t_{j+1}\right)+\psi_{3}(\|x\|)\right]\right\} \\
& +\sum_{j=0}^{m-1}\left[\|p\| \psi_{1}(\|x\|)\left({ }_{t_{j}} I_{g_{j}}^{\alpha_{j}}(1)\right)\left(t_{j+1}\right)+\psi_{2}(\|x\|)\right]+\|p\| \psi_{1}(\|x\|)\left(t_{m} I_{g_{m}}^{\alpha_{m}}(1)\right)(T) \\
& +\sum_{j=0}^{m-1}\left|G_{j+1}\right|\left(\sum_{r=0}^{j}\left[\|p\| \psi_{1}(\|x\|)\left({ }_{t_{r}} I_{g_{r}}^{\alpha_{r}-1}(1)\right)\left(t_{r+1}\right)+\psi_{3}(\|x\|)\right]\right)
\end{aligned}
$$




$$
\begin{aligned}
& =Q_{1}+\left|\lambda_{2}\right| Q_{2}+\|p\| \psi_{1}\left(\rho^{*}\right) Q_{3}+\psi_{2}\left(\rho^{*}\right) Q_{5}+\psi_{3}\left(\rho^{*}\right) Q_{4} \\
& :=Q^{*}
\end{aligned}
$$

which implies that $\|\mathcal{A} x\| \leq Q^{*}$. Thus the set $\mathcal{A} B_{\rho^{*}}$ is uniformly bounded.

In the next step, we will show that $\mathcal{A}$ maps bounded sets into equicontinuous sets of $\operatorname{PC}(J, \mathbb{R})$. Let $\tau_{1}, \tau_{2} \in J_{k}$ for some $k \in\{0,1,2, \ldots, m\}, \tau_{1}<\tau_{2}$. Then, for any $x \in B_{\rho^{*}}$, we have

$$
\begin{aligned}
& \left|\mathcal{A} x\left(\tau_{2}\right)-\mathcal{A} x\left(\tau_{1}\right)\right| \\
& \leq \frac{\left|\lambda_{1}\right|}{|\Omega|}\left|g_{k}\left(\tau_{2}\right)-g_{k}\left(\tau_{1}\right)\right| \\
& \quad+\frac{1}{|\Omega|}\left|g_{k}\left(\tau_{2}\right)-g_{k}\left(\tau_{1}\right)\right|\left\{\left|\lambda_{2}\right|+\|p\| \psi_{1}\left(\rho^{*}\right) \sum_{j=0}^{m} \frac{\left(g_{j}\left(t_{j+1}\right)-g_{j}\left(t_{j}\right)\right)^{\alpha_{j}}}{\Gamma\left(\alpha_{j}+1\right)}\right. \\
& \quad+m \psi_{2}\left(\rho^{*}\right)+\|p\| \psi_{1}\left(\rho^{*}\right) \sum_{j=0}^{m-1}\left|G_{j+1}\right| \sum_{r=0}^{j} \frac{\left(g_{r}\left(t_{r+1}\right)-g_{r}\left(t_{r}\right)\right)^{\alpha_{r}-1}}{\Gamma\left(\alpha_{r}\right)} \\
& \quad+\psi_{3}\left(\rho^{*}\right) \sum_{j=0}^{m-1}\left|G_{j+1}\right|(j+1)+\|p\| \psi_{1}\left(\rho^{*}\right)\left|\beta_{2}\right| \sum_{j=0}^{m} \frac{\left(g_{j}\left(t_{j+1}\right)-g_{j}\left(t_{j}\right)\right)^{\alpha_{j}-1}}{\Gamma\left(\alpha_{j}\right)} \\
& \left.\quad+\left|\beta_{2}\right| m \psi_{3}\left(\rho^{*}\right)\right\}+\|p\| \psi_{1}\left(\rho^{*}\right)\left|\frac{\mid\left(g_{k}\left(\tau_{2}\right)-g_{k}\left(t_{k}\right)\right)^{\alpha_{k}}}{\Gamma\left(\alpha_{k}+1\right)}-\frac{\left(g_{k}\left(\tau_{1}\right)-g_{k}\left(t_{k}\right)\right)^{\alpha_{k}}}{\Gamma\left(\alpha_{k}+1\right)}\right| .
\end{aligned}
$$

As $\tau_{1} \rightarrow \tau_{2}$, the right-hand side of this inequality tends to zero independently of unknown variable $x$. Therefore by the Arzelá-Ascoli theorem it follows that the operator $\mathcal{A}: \operatorname{PC}(J, \mathbb{R}) \rightarrow \operatorname{PC}(J, \mathbb{R})$ is completely continuous.

Finally, we show that there exists an open set $U \subseteq \operatorname{PC}(J, \mathbb{R})$ with $x \neq \lambda \mathcal{A}(x)$ for $\lambda \in(0,1)$ and $x \in \partial U$.

Let $x \in \operatorname{PC}(J, \mathbb{R})$ and $x=\lambda \mathcal{A}(x)$ for some $0<\lambda<1$. Thus, for each $t \in J$, we have

$$
\begin{aligned}
|x(t)|= & \lambda|\mathcal{A} x(t)| \\
\leq & \frac{\left|\lambda_{1}\right|}{|\Omega|}\left(\left|\beta_{2}\right|+\left(\sum_{j=0}^{m}\left|G_{j}\right|\right)+\left(\sum_{j=0}^{k}\left|G_{j}\right|\right)\right) \\
& +\frac{1}{|\Omega|}\left(\left|\beta_{1}\right|+\left(\sum_{j=0}^{k}\left|G_{j}\right|\right)\right)\left\{\left|\lambda_{2}\right|+\sum_{j=0}^{m-1}\left[\left(t_{j} I_{g_{j}}^{\alpha_{j}}\left|f_{x}\right|\right)\left(t_{j+1}\right)+\left|\phi_{j+1}\left(x\left(t_{j+1}\right)\right)\right|\right]\right. \\
& +\left(t_{m} I g_{m}^{\alpha_{m}}\left|f_{x}\right|\right)(T)+\sum_{j=0}^{m-1}\left|G_{j+1}\right|\left(\sum_{r=0}^{j}\left[\left(t_{r} I_{g_{r}}^{\alpha_{r}-1}\left|f_{x}\right|\right)\left(t_{r+1}\right)+\left|\phi_{r+1}^{*}\left(x\left(t_{r+1}\right)\right)\right|\right]\right) \\
& \left.+\left|\beta_{2}\right|\left(_{t_{m}} I_{g_{m}}^{\alpha_{m}-1}\left|f_{x}\right|\right)(T)+\left|\beta_{2}\right| \sum_{j=0}^{m-1}\left[\left(t_{j} I_{g_{j}}^{\alpha_{j}-1}\left|f_{x}\right|\right)\left(t_{j+1}\right)+\left|\phi_{j+1}^{*}\left(x\left(t_{j+1}\right)\right)\right|\right]\right\} \\
& +\sum_{j=0}^{k-1}\left[\left(t_{t_{j}} I_{g_{j}}^{\alpha_{j}}\left|f_{x}\right|\right)\left(t_{j+1}\right)+\left|\phi_{j+1}\left(x\left(t_{j+1}\right)\right)\right|\right]+\left(t_{k} I_{g_{k}}^{\alpha_{k}}\left|f_{x}\right|\right)(t)
\end{aligned}
$$




$$
\begin{aligned}
& +\sum_{j=0}^{k-1}\left|G_{j+1}\right|\left(\sum_{r=0}^{j}\left[\left({ }_{t r} I_{g_{r}}^{\alpha_{r}-1}\left|f_{x}\right|\right)\left(t_{r+1}\right)+\left|\phi_{r+1}^{*}\left(x\left(t_{r+1}\right)\right)\right|\right]\right) \\
\leq & \left(Q_{1}+\left|\lambda_{2}\right| Q_{2}\right)+\left(\|p\| \psi_{1}(\|x\|) Q_{3}+\psi_{2}(\|x\|) Q_{5}+\psi_{3}(\|x\|) Q_{4}\right),
\end{aligned}
$$

which can be expressed as

$$
\frac{\|x\|}{\left(Q_{1}+\left|\lambda_{2}\right| Q_{2}\right)+\left(\|p\| \psi_{1}(\|x\|) Q_{3}+\psi_{2}(\|x\|) Q_{5}+\psi_{3}(\|x\|) Q_{4}\right)} \leq 1 .
$$

In view of $\left(H_{5}\right)$, there exists $M$ such that $\|x\| \neq M$. Let

$$
U=\{x \in P C(J, \mathbb{R}):\|x\|<M\} .
$$

Note that the operator $\mathcal{A}: \bar{U} \rightarrow \mathrm{PC}(J, \mathbb{R})$ is continuous and completely continuous. From the choice of $U$ there is no $x \in \partial U$ such that $x=\lambda \mathcal{A}(x)$ for some $\lambda \in(0,1)$. Consequently, by the nonlinear alternative of Leray-Schauder type (Lemma 3.3) we deduce that $\mathcal{A}$ has a fixed point $x \in \bar{U}$, which is a solution of problem (1.1). This completes the proof.

In the particular case $p(t)=1$ and $\psi_{i}(\|x\|)=\Lambda_{i}\|x\|+N_{i}, i=1,2,3$, we have the following corollary.

Corollary 3.5 Let $f: J \times \mathbb{R} \rightarrow \mathbb{R}$ and $\phi_{k}, \phi_{k}^{*}: \mathbb{R} \rightarrow \mathbb{R}, k=1,2, \ldots, m$, be continuous functions. Assume that:

$\left(A_{1}\right)$ There exist constants $\Lambda_{i}, N_{i}>0, i=1,2,3$, such that

$$
\begin{aligned}
& |f(t, x)| \leq \Lambda_{1}\|x\|+N_{1} \quad \text { for all } t \in J, x \in \mathbb{R}, \\
& \left|\phi_{k}(x)\right| \leq \Lambda_{2}\|x\|+N_{2}, \quad\left|\phi_{k}^{*}\right| \leq \Lambda_{3}\|x\|+N_{3} \quad \text { for all } x \in \mathbb{R}, k=1,2, \ldots, m .
\end{aligned}
$$

If

$$
\Lambda_{1} Q_{3}+\Lambda_{2} Q_{5}+\Lambda_{3} Q_{4}<1,
$$

then the impulsive fractional boundary value problem (1.1) has at least one solution on J.

The result for nonimpulse effects is given by the following:

Corollary 3.6 Assume that condition $\left(H_{3}\right)$ holds. In addition, we suppose that:

$\left(B_{1}\right)$ There exists a constant $M>0$ such that

$$
\frac{M}{Q_{1}^{*}+\left|\lambda_{2}\right| Q_{2}^{*}+\|p\| \psi_{1}(M) Q_{3}^{*}}>1
$$

Then boundary value problem (3.2) has at least one solution on J.

In addition, suppose that $\left(A_{1}\right)$ holds with $m=0$ (i.e., $\left.\phi_{k}(\cdot)=\phi_{k}^{*}(\cdot) \equiv 0\right)$ and that $\Lambda_{1} Q_{3}^{*}<1$.

Then problem (2.7) has at least one solution on J. 


\section{Examples}

In this section, we present some examples to illustrate our results.

Example 4.1 Consider the following impulsive boundary value problem containing $\mathrm{Ca}$ puto fractional derivative with respect to another function of the form

$$
\left\{\begin{array}{l}
t_{k} D_{\left(e^{t}+t^{k}\right)}^{\left(\frac{2 k+3}{k+3}\right)} x(t)=f(t, x(t)), \quad t \neq t_{k}=k, k=0,1,2,3, \\
x\left(t_{k}^{+}\right)-x\left(t_{k}^{-}\right)=\phi_{k}\left(x\left(t_{k}\right)\right), \quad k=1,2,3, \\
D_{\left(e^{t}+t^{k}\right)} x\left(t_{k}^{+}\right)-D_{\left(e^{t}+t^{k-1}\right)} x\left(t_{k}^{-}\right)=\phi_{k}^{*}\left(x\left(t_{k}\right)\right), \quad k=1,2,3, \\
x(0)+\frac{2}{3}\left(D_{\left(e^{t}+1\right)} x\right)(0)=\frac{1}{2}, \quad x(4)+\frac{3}{5}\left(D_{\left(e^{t}+t^{3}\right)} x\right)(4)=\frac{5}{7} .
\end{array}\right.
$$

Here $\alpha_{k}=(2 k+3) /(k+2), g_{k}(t)=e^{t}+t^{k}, k=0,1,2,3, m=3, T=4, \beta_{1}=2 / 3, \beta_{2}=3 / 5$, $\lambda_{1}=1 / 2$, and $\lambda_{2}=5 / 7$. Then we can compute that $\Omega \approx 96.53148336, Q_{1} \approx 1.003798415$, $Q_{2} \approx 1.007596831, Q_{3} \approx 5114.088162, Q_{4} \approx 514.9585869$, and $Q_{5} \approx 6.022790493$.

(i) Let $f:[0,4] \times \mathbb{R} \rightarrow \mathbb{R}, \phi_{k}: \mathbb{R} \rightarrow \mathbb{R}$, and $\phi_{k}^{*}: \mathbb{R} \rightarrow \mathbb{R}$ be the functions defined by

$$
\begin{aligned}
& f(t, x)=\frac{\cos ^{2} \pi t}{2(t+100)^{2}}\left(\frac{x^{2}+2|x|}{|x|+1}\right)+\frac{3}{2}, \\
& \phi_{k}(x)=\frac{1}{(k+20)} \sin |x|+\frac{1}{3}, \quad k=1,2,3, \\
& \phi_{k}^{*}(x)=\frac{1}{(k+60)^{2}} \tan ^{-1}|x|+\frac{2}{5}, \quad k=1,2,3 .
\end{aligned}
$$

It follows that $|f(t, x)-f(t, y)| \leq(1 / 100)^{2}|x-y|,\left|\phi_{k}(x)-\phi_{k}(y)\right| \leq(1 / 21)|x-y|$, and $\mid \phi_{k}^{*}(x)-$ $\phi_{k}^{*}(y)\left|\leq(1 / 61)^{2}\right| x-y \mid$ with $L_{1}=(1 / 100)^{2}, L_{2}=1 / 21$, and $L_{3}=(1 / 61)^{2}$. Therefore we obtain $L_{1} Q_{3}+L_{2} Q_{5}+L_{3} Q_{4} \approx 0.9366008889<1$. Hence by Theorem 3.1 we get that problem (4.1) with $f(t, x), \phi_{k}(x)$, and $\phi_{k}^{*}(x)$ defined in (4.2) has a unique solution on $[0,4]$.

(ii) Now we consider the functions $f:[0,4] \times \mathbb{R} \rightarrow \mathbb{R}, \phi_{k}: \mathbb{R} \rightarrow \mathbb{R}$, and $\phi_{k}^{*}: \mathbb{R} \rightarrow \mathbb{R}$ defined by

$$
\begin{aligned}
f(t, x) & =\frac{e^{-t}}{5(t+40)}\left(\frac{1}{900} x^{2}+\frac{1}{150}\right), \\
\phi_{k}(x) & =\frac{1}{800} x^{2}+\frac{1}{k+19}, \quad k=1,2,3, \\
\phi_{k}^{*}(x) & =\frac{1}{9000} x^{2}+\frac{1}{(39+k)^{2}}, \quad k=1,2,3 .
\end{aligned}
$$

Setting $p(t)=1 /(5(t+40)), \psi_{1}(x)=(1 / 900) x^{2}+(1 / 150), \psi_{2}(x)=(1 / 800) x^{2}+(1 / 20)$, and $\psi_{3}(x)=(1 / 9000) x^{2}+(1 / 1600)$, conditions $\left(H_{3}\right)-\left(H_{4}\right)$ are fulfilled. From $\|p\|=1 / 200$ by direct computing we find that there exists a constant $M \in(4.029734051,6.704750535)$ satisfying condition $\left(H_{5}\right)$ in Theorem 3.4. By Theorem 3.4 problem (4.1) with all functions defined in (4.3) has at least one solution in $[0,4]$. 
(iii) Let us consider the functions $f:[0,4] \times \mathbb{R} \rightarrow \mathbb{R}, \phi_{k}: \mathbb{R} \rightarrow \mathbb{R}$, and $\phi_{k}^{*}: \mathbb{R} \rightarrow \mathbb{R}$ defined by

$$
\begin{aligned}
& f(t, x)=\left(\frac{1}{(130+t)^{2}}\right) \frac{x^{8} e^{-x^{2}}}{|x|^{7}+1}+\tan ^{-1}|x|+2, \\
& \phi_{k}(x)=\left(\frac{1}{19+k}\right) \frac{x^{10} \cos ^{2}|x|}{|x|^{9}+3}+\frac{1}{2} \sin ^{4}|x|+1, \quad k=1,2,3, \\
& \phi_{k}^{*}(x)=\left(\frac{1}{(39+k)^{2}}\right) \frac{|x|^{5} \sin ^{2}|x|}{x^{4}+5}+\frac{1}{4} e^{-|x|} \quad k=1,2,3 .
\end{aligned}
$$

It is obvious that $|f(t, x)| \leq(1 / 16900)|x|+((\pi / 2)+2),\left|\phi_{k}(x)\right| \leq(1 / 20)|x|+(3 / 2)$, and $\left|\phi_{k}^{*}(x)\right| \leq(1 / 1600)|x|+(1 / 4)$, so that condition $A_{1}$ is satisfied. Further, by directly computation we have $\Lambda_{1} Q_{3}+\Lambda_{2} Q_{5}+\Lambda_{3} Q_{4} \approx 0.9255974084<1$. Then from Corollary 3.5 it follows that problem (4.1) with nonlinear functions in (4.4) has at least one solution in $[0,4]$.

\section{Discussion}

Problem (1.1) can be extended to impulsive fractional differential equations with delays by replacing the function $f(t, x(t))$ with $f(t, x(t), x(\theta(t)))$, where $\theta: J \rightarrow J$ and $\theta(t) \leq t$. Next, we give the existence theorems without proofs for impulsive delay fractional differential equations with boundary conditions of the form

$$
\left\{\begin{array}{l}
t_{k} D_{g_{k}}^{\alpha_{k}} x(t)=f(t, x(t), x(\theta(t))), \quad t \neq t_{k}, k=0,1,2, \ldots, m, \\
x\left(t_{k}^{+}\right)-x\left(t_{k}^{-}\right)=\phi_{k}\left(x\left(t_{k}\right)\right), \quad k=1,2, \ldots, m, \\
D_{g_{k}} x\left(t_{k}^{+}\right)-D_{g_{k-1}} x\left(t_{k}^{-}\right)=\phi_{k}^{*}\left(x\left(t_{k}\right)\right), \quad k=1,2, \ldots, m, \\
x(0)+\beta_{1} D_{g_{0}} x(0)=\lambda_{1}, \quad x(T)+\beta_{2} D_{g_{m}} x(T)=\lambda_{2} .
\end{array}\right.
$$

Theorem 5.1 Let $g_{k} \in C^{2}([0, T])$ with $g_{k}^{\prime}(t)>0$ for $t \in[0, T], k=0,1, \ldots$, m. Suppose that the function $f: J \times \mathbb{R}^{2} \rightarrow \mathbb{R}$ satisfies

$\left(H_{6}\right)|f(t, x, u)-f(t, y, v)| \leq L_{1}|x-y|+L_{4}|u-v|, L_{1}, L_{4}>0, \forall t \in J, x, y, u, v \in \mathbb{R}$.

In addition, assume that the functions $\phi_{k}: \mathbb{R} \rightarrow \mathbb{R}$ and $\phi_{k}^{*}: \mathbb{R} \rightarrow \mathbb{R}, k=1,2, \ldots, m$, satisfy $\left(H_{2}\right)$.

If

$$
\left(L_{1}+L_{4}\right) Q_{3}+L_{2} Q_{5}+L_{3} Q_{4}<1,
$$

then the boundary value problem of impulsive delay fractional differential equations with respect to other functions (5.1) has a unique solution on J.

Theorem 5.2 Assume that the functions $\phi_{k}$ and $\phi_{k}^{*}$ satisfy $\left(H_{4}\right)$ for $k=1,2, \ldots$, m and suppose that

$\left(H_{7}\right)$ There exist two continuous nondecreasing functions $\psi_{1}, \psi_{4}:[0, \infty) \rightarrow(0, \infty)$ and two continuous functions $p_{1}, p_{2}: J \rightarrow \mathbb{R}^{+}$such that

$$
|f(t, x, y)| \leq p_{1}(t) \psi_{1}(|x|)+p_{2}(t) \psi_{4}(|y|), \quad(t, x, y) \in J \times \mathbb{R}^{2} .
$$


$\left(H_{8}\right)$ There exists a constant $N>0$ such that

$$
\frac{N}{\left(Q_{1}+\left|\lambda_{2}\right| Q_{2}\right)+\left[\left\{\left\|p_{1}\right\| \psi_{1}(N)+\left\|p_{2}\right\| \psi_{4}(N)\right\} Q_{3}+\psi_{2}(N) Q_{5}+\psi_{3}(N) Q_{4}\right]}>1 .
$$

Then the impulsive fractional boundary value problem with delay (5.1) has at least one solution on $J$.

\section{Conclusion}

In this paper, we initiate the study of existence and uniqueness of solutions for a new class of impulsive fractional boundary value problems with separated boundary conditions containing the Caputo fractional derivative of a function with respect to another function. The existence of solutions is established by using the Leray-Schauder nonlinear alternative, whereas the uniqueness result is proved via Banach's contraction mapping principle. Examples are also constructed to illustrate the main results. The considered problem generates many types and also mixed types of impulsive fractional differential equations with boundary conditions.

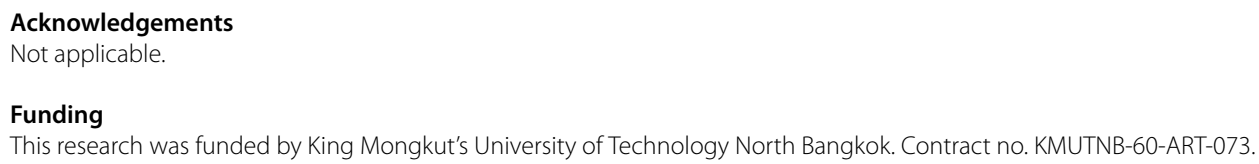

\section{Author details}

${ }^{1}$ Intelligent and Nonlinear Dynamic Innovations Research Center, Department of Mathematics, Faculty of Applied Science, King Mongkut's University of Technology North Bangkok, Bangkok, Thailand. ²Department of Mathematics Statistic and Computer Science, Faculty of Liberal Arts and Science, Kasetsart University, Nakhonpathom, Thailand. ${ }^{3}$ Department of Mathematics, University of loannina, loannina, Greece. ${ }^{4}$ Nonlinear Analysis and Applied Mathematics (NAAM)-Research Group, Department of Mathematics, Faculty of Science, King Abdulaziz University, Jeddah, Saudi Arabia.

\section{Publisher's Note}

Springer Nature remains neutral with regard to jurisdictional claims in published maps and institutional affiliations.

Received: 14 August 2019 Accepted: 13 November 2019 Published online: 21 November 2019

\section{References}

1. Benchohra, M., Henderson, J., Ntouyas, S.K.: Impulsive Differential Equations and Inclusions, vol. 2. Hindawi Publishing Corporation, New York (2006)

2. Bohner, M., Heidarkhani, S., Salari, A., Caristi, G.: Existence of three solutions for impulsive multi-point boundary value problems. Opusc. Math. 37, 353-379 (2017)

3. Shen, J., Wang, W.: Impulsive boundary value problems with nonlinear boundary conditions. Nonlinear Anal. TMA 69, 4055-4062 (2008)

4. Wang, J., Zhou, Y., Feckan, M.: On recent developments in the theory of boundary value problems for impulsive fractional differential equations. Comput. Math. Appl. 64, 3008-3020 (2012)

5. Liu, Y.: Solvability of impulsive periodic boundary value problems for higher order fractional differential equations. Arab. J. Math. 6, 195-214 (2016)

6. Thaiprayoon, Ch., Tariboon, J., Ntouyas, S.K.: Impulsive fractional boundary-value problems with fractional integral jump conditions. Bound. Value Probl. 2014, Article ID 17 (2014)

7. Asma, Ali, A., Shah, K., Jarad, F.: Ulam-Hyers stability analysis to a class of nonlinear implicit impulsive fractional differential equations with three point boundary conditions. Adv. Differ. Equ. 2019, Article ID 7 (2019)

8. Bai, Z., Dong, X., Yin, C.: Existence results for impulsive nonlinear fractional differential equation with mixed boundary conditions. Bound. Value Probl. 2016, Article ID 63 (2016) 
9. Kilbas, A.A., Srivastava, H.M., Trujillo, J.J.: Theory and Applications of Fractional Differential Equations. North-Holland Mathematics Studies, vol. 204. Elsevier, Amsterdam (2006)

10. Almeida, R.: A Caputo fractional derivative of a function with respect to another function. Commun. Nonlinear Sci. Numer. Simul. 44, 460-481 (2017)

11. Almeida, R., Malinowska, A., Monteiro, T.: Fractional differential equations with a Caputo derivative with respect to a kernel function and their applications. Math. Methods Appl. Sci. 41, 336-352 (2018)

12. Ameen, R., Jarad, F., Abdeljawad, T.: Ulam stability for delay fractional differential equations with a generalized Caputo derivative. Filomat 32, 5265-5274 (2018)

13. Samet, B., Aydi, H.: Lyapunov-type inequalities for an anti-periodic fractional boundary value problem involving $\psi$-Caputo fractional derivative. J. Inequal. Appl. 2018, Article ID 286 (2018)

14. Katugampola, U.: A new approach to generalized fractional derivatives. Bull. Math. Anal. Appl. 6, 1-15 (2014)

15. Wang, G.: Explicit iteration and unbounded solutions for fractional integral boundary value problem on an infinite interval. Appl. Math. Lett. 47, 1-7 (2015)

16. Wang, G., Pei, K., Agarwal, R.P., Zhang, L., Ahmad, B.: Nonlocal Hadamard fractional boundary value problem with Hadamard integral and discrete boundary conditions on a half-line. J. Comput. Appl. Math. 343, 230-239 (2018)

17. Wang, G., Pei, K., Chen, Y.: Stability analysis of nonlinear Hadamard fractional differential system. J. Franklin Inst. 356, 6538-6546 (2019)

18. Tariboon, J., Ntouyas, S.K.: Initial and boundary value problems via exponential fractional calculus. Int. Electron. J. Pure Appl. Math. 12(1), 1-19 (2018)

19. Ntouyas, S.K., Tariboon, J., Sawaddee, C.: Nonlocal initial and boundary value problems via fractional calculus with exponential singular kernel. J. Nonlinear Sci. Appl. 11, 1015-1030 (2018)

20. Granas, A., Dugundji, J.: Fixed Point Theory. Springer, New York (2005)

\section{Submit your manuscript to a SpringerOpen ${ }^{\circ}$ journal and benefit from:}

- Convenient online submission

- Rigorous peer review

Open access: articles freely available online

- High visibility within the field

- Retaining the copyright to your article

Submit your next manuscript at $\boldsymbol{\nabla}$ springeropen.com 